\title{
INTEGRAL OPERATORS IN THE THEORY OF INDUCED BANACH REPRESENTATIONS II. THE BUNDLE APPROACH
}

\author{
I.E. SCHOCHETMAN \\ Mathematics Department, Oakland University \\ Rochester, Michigan 48603 \\ (Received March 4, 1981)
}

ABSTRACT. Let $\mathrm{G}$ be a locally compact group, $\mathrm{H}$ a closed subgroup and $\mathrm{L}$ a Banach representation of $\mathrm{H}$. Suppose $\mathrm{U}$ is a Banach representation of $\mathrm{G}$ which is induced by $\mathrm{L}$. Here, we continue our program of showing that certain operators of the integrated form of $U$ can be written as integral operators with continuous kernels. Specifically, we show that: (1) the representation space of a Banach bundle; (2) the above operators become integral operators on this space with kernels which are continuous crosssections of an associated kernel bundle.

KEY WURDS AND PHRASES. LOcalxy compact group, Banach representation, induced representation, integrated form, integrak operator, vector field, cross-section, continuity structure, banach bundle, quotient topology, kernel bundle.

1980 MATHEMATICS SUBJELT CLASSIFICATION CODE. Primary: 22020, 22030, 47G05, 34A10 Secondary: $22012,28 B 99,46 B 99,54 B 15$.

\section{INTRODUCTION.}

Let $\mathrm{G}$ be a locally compact group with right Haar measure $\mathrm{dx}, \mathrm{H}$ a closed subgroup of $G$ with right Haar measure $d t$, and $\pi: G \rightarrow X$, the canonical projection onto the right coset space $X=G / H$. Suppose $L$ is a (strongly continuous) representation of $H$ on the Banach space $E$. Suppose also that $U$ is a representation of $G$ on a certain Banach space $F_{U}$ which is induced by $L$ in the sense of [1, sec. 3]. The integrated form of $U$ is the representation of $L^{1}(G)$ determined by the bounded operators $U(\varphi)$ on $F_{U}$, where

$$
U(\varphi)=\int_{G} \varphi(x) U(x) d x
$$


for $\varphi$ a continuous function on $G$ with compact support, i.e. $\varphi \varepsilon C_{C}(G)$. It is well-known [2] that these operators can be written as integral operators with continuous kernels in the following sense: for $f$ in a certain dense subspace of $F_{U}$, we have

$$
U(\varphi) f(x)=\int_{X} I_{\varphi}(x, y) f(y) d(\pi(y)), x \in G,
$$

where $d(\pi(y))$ is a quasi-invariant measure on $X$ and $I_{\varphi}$ is a continuous mapping of $G \times G$ into the bounded operators $\operatorname{Hom}(E)$ on $E$. The existence of the integral is determined by the fact that for each $x$ in $G$, the mapping $I_{\varphi}(x, \cdot) f(\cdot)$ is constant on cosets. Thus, the kernel $\mathrm{I}_{\varphi}$ for $\mathrm{U}(\varphi)$ is defined on $G \times G$, while the integration is over $\mathrm{X}$. Moreover, the mapping $\mathrm{I}_{\varphi}$ is not constant on cosets in general.

Our primary objective in [2] was to represent the operators $U(\varphi)$ as integral operators with continuous kernels in a consistent fashion, i.e. where the kernels and integration are defined over the same space. There are two canonical choices for this space -- namely G and X . In Chapter II of [2] we acomplished our objective over each of these spaces.

In section 3 of [2], we constructed a representation $V$ of $G$ on a Banach function space $F_{V}$ which was isometrically equivalent to $U$. In particular, each operator $V(\varphi)$ was written in the following form: for each continuous $g$ in a certain dense subspace of $F_{V}$, we have

$$
V(\varphi) g(x)=\int_{G} J_{\varphi}(x, y) g(y) d y, x \in G,
$$

where $\mathrm{J}_{\varphi}$ is a continuous mapping from $\mathrm{G} \times \mathrm{G}$ into Hom(E). Although this result is satisfactory from the consistency viewpoint, there is a significant shortcoming. The space $F_{V}$ is a proper closed subspace of a vector-valued ${ }_{L}{ }^{P}$-space. Thus, many of the important existing results for integral operators cannot be used with this model of the integrated form of $U$.

In section 4 of [2], we next turned our attention to the quotient space $X$. We constructed a representation $W$ of $G$ on a continuous sum $F_{W}$ of Banach spaces $\left\{E_{\xi}: \xi \in X\right\}$ which was also isometrically equivalent to $U$. Each operator $W(\varphi)$ 
was written as follows: for each "continuous" vector field $h$ in a certain dense subspace of $F_{W}$, we have

$$
W(\varphi) h(\xi)=\int_{X} K_{\varphi}(\xi, \eta) h(\eta) d \eta, \xi \varepsilon X,
$$

where $\mathrm{K}_{\varphi}$ is a "continuous" field of bounded operators in $\Pi$ Hom $\left(E_{\eta}, E_{\xi}\right)$ and $\mathrm{K}_{\varphi}(\xi, \eta) \varepsilon \operatorname{Hom}\left(\mathrm{E}_{\eta}, \mathrm{E}_{\xi}\right)$. This model requires the knowledge of continuity structures and $L^{P}$-theory for vector fields $[3,4]$, as well as the theory of kernels and integral operators for such Lebesque spaces [4]. (The latter was developed by the author expressly for this purpose.) Since the space $F_{W}$ is a full $L^{p}$-space, this model of $\mathrm{U}$ does not have the shortcoming that $\mathrm{V}$ has. In fact, it proved to be quite useful in studying certain compactness properties of the integrated form of $U$ [ 2 , Ch. IV] . However, $W$ has two minor shortcomings which are more akin to mathematical utility and aesthetics than to mathematical substance. First, a continuity structure (and its implications) is quite complicated and is a less intuitive object to work with than is the more fundamental and familiar notion of topological continuity. Second, although the kernels $K_{\varphi}$ do belong to a continuity structure in $\Pi H_{0}\left(E_{\eta}, E_{\xi}\right)$, this structure partially loses a desirable property in the transition from $G$ to $X$. (See pp. 25-29 of [2] for a rigorous explanation.)

Since the writing of [2], it has been discovered [5] that the theory of continuity structures is equivalent to the theory of Banach bundles [6]. Moreover, in the bundle context, the appropriate mappings are cross-sections, so that continuity is simply topological continuity. These facts suggest:

(1) The representation $W$ can be reconstructed in the setting of Banach bundles.

(2) The kernels $\mathrm{K}_{\varphi}$ should be continuous cross-sections for a suitable bundle.

The main objectives of this paper are to show exactly how to accomplish (1) and (2). Section 2 is devoted to recalling the necessary preliminaries. In sections 3 and 4 , we construct the Banach bundles corresponding to the continuity structures in $\Pi E_{\xi}$ and $\Pi \operatorname{Hom}\left(E_{\eta}, E_{\xi}\right)$ respectively. Finally, in section 5, we show that the kernels $\mathrm{K}_{\varphi}$ are continuous cross-sections for the bundle of section 4 . 


\section{PRELIMINARIES.}

The following is a brief summary of sections 1 and 2 of [2].

Let $\Delta_{G}$ and $\Delta_{H}$ be the modular functions for $G$ and $H$ respectively. The quotient function $\Delta_{H} /\left(\Delta_{G} \mid H\right)$ is a continuous homomorphism of $H$ into the positive reals which we shall denote by $\delta$. Let $\rho$ be a continuous, non-negative function on $G$ satisfying $\rho(t x)=\delta(t) \rho(x), t \varepsilon H, x \in G$. Also let $d \xi=d(\pi(x)$ ) be the quasi-invariant measure on $\mathrm{X}$ corresponding to $\rho$.

The representation $L$ of $H$ on $E$ is "Banach inducible up to $G$ " if there exists a pair $(p, q)$ such that $1 \leq p \leq \infty, 0<q \leq \infty$ and

$$
\delta(t)^{1 / q}\|L(t)\| \leq b \delta(t)^{1 / p}, \quad t \varepsilon H,
$$

for some $b \geq 1$. Given such a pair, we can construct [1] an isometric representation of $G$ on a certain Banach function space as follows. Let $C_{q}(G, L)$ denote the linear space of all continuous mappings $f: G \rightarrow E$ such that: (i) $f$ has compact support modulo H, i.e. $\pi(\operatorname{supp}(f))$ is compact. (ii) $f$ is $(L, q)$-homogeneous, i.e.

$$
f(t x)=\delta(t)^{1 / q_{L}(t) f(x), t} \varepsilon H, x \in G .
$$

For a suitable $\mathrm{L}^{\mathrm{P}}$-norm on $\mathrm{C}_{\mathrm{q}}(\mathrm{G}, \mathrm{L}) \quad[1$, sec. 3], we find that right translation $f \rightarrow f x$ by an element $x$ of $G$ is an isometry. Hence, its extension to the completion $F_{U}$ of $C_{q}(G, L)$ is also an isometry which we denote by $U(x)$. The resulting mapping $U$ is then an isometric representation of $G$ on $F_{U}$ which we call the induced Banach representation corresponding to $(L, p, q)$. We refer the reader to

[1] for a thorough development of such representations.

In this setting, the operators $U(\varphi), \varphi \in C_{C}(G)$, may be written as follows:

$U(\varphi) f(x)=\Delta_{G}(x)^{-1} \int_{X} \rho(y)^{-1}\left[\int_{H} \delta(t)^{q: 1} \varphi\left(x^{-1} t y\right) L(t)\right] d t f(y) d(\pi(y))$,

for $f$ in $\mathrm{C}_{\mathrm{q}}(\mathrm{G}, \mathrm{L})$ (where $\mathrm{q}: \mathrm{p}=1 / \mathrm{q}-1 / \mathrm{p}$ ). Thus, the mapping $\mathrm{I}_{\varphi}$ referred to in the introduction is given by

$$
I_{\varphi}(x, y)=\Delta_{G}(x)^{-1} \rho(y)^{-1} \int_{H} \delta(t)^{q: 1}{ }_{\varphi}\left(x^{-1} t y\right) L(t) d t, x, y \in G .
$$


Before concluding this section, observe that if we let $M=\delta^{\mathrm{q}: \mathrm{p}_{L}}$, then $M$ is a bounded representation of $H$ on $E$ with bound $b$. Hence, we may renorm $E$ by defining

$$
\|v\|_{M}=\sup \{\|M(t) v\|: t \varepsilon H\}, \quad v \varepsilon E
$$

We thus obtain a Banach space $E_{M}$ which is equivalent to $E$ since

$$
\|\mathrm{v}\| \leq\|\mathrm{v}\|_{\mathrm{M}} \leq \mathrm{b}\|\mathrm{v}\|, \quad \mathrm{v} \in \mathrm{E}
$$

The Banach spaces $\operatorname{Hom}(E)$ and $\operatorname{Hom}\left(\mathrm{E}_{M}\right)$ are also equivalent with

$$
\|\mathrm{T}\| \leq\|\mathrm{T}\|_{\mathrm{M}} \leq \mathrm{b}\|\mathrm{T}\|, \mathrm{T} \varepsilon \operatorname{Hom}(\mathrm{E})
$$

The following result will be useful in what follows:

LEMMA 2.1. The mapping $(t, v) \rightarrow L(t) v$ of $H \times E$ into $E$ is continuous.

PROOF. This follows from the fact that $L$ is strongly continuous and locally bounded $[1,3 \cdot 3]$

It will also be convenient to $\mathrm{fix}$ in advance a compact neighborhood $\mathrm{Z}$ of the identity $e$ in $G$ for use later on.

\section{THE VECTOR FIELD BUNDLE}

In order to construct the bundle version of $W$, we begin as in section 4 of [2]

Consider the space $G \times E$ with equivalence relation $\sim$ defined as follows: if $\mathrm{x}, \mathrm{y} \in \mathrm{G}$ and $\mathrm{v}, \mathrm{w} \varepsilon \mathrm{E}$, then $(\mathrm{x}, \mathrm{v}) \sim(\mathrm{y}, \mathrm{w})$ if there exists $\mathrm{t}$ in $\mathrm{H}$ such that $y=t x$ and $w=L(t) v$. Denote the resulting space of equivalence classes (with quotient topology) by $E$ and let $\sigma: G \times E \rightarrow E$ be the canonical projection. Also let $\tau: E \rightarrow X$ be the (well-defined) projection given by $\tau(\sigma(x, v))=\pi(x)$. We then have the following composition:

$$
\mathrm{G} \times \mathrm{E} \stackrel{\sigma}{\rightarrow} \mathrm{i} \stackrel{\mathrm{X}}{\rightarrow}
$$

LEMMA 3.1. The mapping $\sigma$ is continuous and open.

PROOF. The openness of $\sigma$ follows from the fact that the saturation ${ }^{-1}(\sigma(A \times B))$ of a basic open subset $A \times B$ of $G \times E$ is of the form 


$$
U\{(t A) \times(L(t) B): T \in H\},
$$

which is open in $G \times E$.

LEMMA 3.2. The mapping $\tau$ is continuous and open.

PROOF. The continuity and openness of $\tau$ follow from the continuity and openness of $\sigma$ and $\pi$.

PROPOSITION 3.3. The space $E$ is Hausdorff.

PROOF. Let $\left\{\theta_{i}\right\}$ be a net in $E$ converging to $\theta$ and $\theta^{\prime}$ in $E$, where $\theta=\sigma(x, v)$ and $\theta^{\prime}=\sigma\left(x^{\prime}, v^{\prime}\right)$. Since $\sigma$ is open, passing to a subnet if necessary, we may assume there exists a corresponding net $\left\{\left(\mathrm{x}_{i}, \mathrm{v}_{\mathbf{i}}\right)\right\}$ in $\mathrm{G} \times \mathrm{E}$ such that $\sigma\left(x_{i}, v_{i}\right)=\theta_{i}, i=1,2$, and $\left(x_{i}, v_{i}\right) \rightarrow(x, v)$ in $G \times E$. Similarly, we may assume there exists a corresponding net $\left\{\left(x_{i}^{\prime}, v_{i}^{\prime}\right)\right\}$ in $G \times E$ such that $\sigma\left(x_{i}^{\prime}, v_{i}^{\prime}\right)=\theta_{i}$ and $\left(x_{i}^{\prime}, v_{i}^{\prime}\right) \rightarrow\left(x^{\prime}, v^{\prime}\right)$. Since $\sigma\left(x_{i}, v_{i}\right)=\sigma\left(x_{i}^{\prime}, v_{i}^{\prime}\right)$, there exists $t_{i} \varepsilon H$ such that $x_{i}^{\prime}=t_{i} x_{i}$ and $v_{i}^{\prime}=L\left(t_{i}\right) v_{i}$. Consequently, $t=x^{\prime} x^{-1}$ is an element of $H$ since the net $\left\{t_{i}=x_{i}^{\prime} x_{i}^{-1}\right\}$ converges to $x^{\prime} x^{-1}$ and $H$ is closed in G. Moreover, by 1.1 and the triangle inequality, we see that $v^{\prime}=L(t) v$. Thus, $\theta=\theta^{\prime}$ and $E$ is Hausdorff.

The next step is to make $(E, X, \tau)$ into a Banach bundle. As on p. 12 of [2], define $E_{\xi}=\tau^{-1}(\xi), \xi \varepsilon X$. For fixed $x$ in $" \xi=\pi^{-1}(\xi)$ ", each element of $E_{\xi}$ is uniquely of the form $\sigma(x, v), v \in E$. Thus, $E_{\xi}$ becomes a Banach space equivalent to $E$ and $E_{M}$ under the following (well-defined) operations:

$$
\begin{aligned}
& \sigma(\mathrm{x}, \mathrm{v})+\sigma(\mathrm{x}, \mathrm{w})=\sigma(\mathrm{x}, \mathrm{v}+\mathrm{w}) \\
& \operatorname{co}(\mathrm{x}, \mathrm{v})=\sigma(\mathrm{x}, \mathrm{cv}) \\
& \|\sigma(\mathrm{x}, \mathrm{v})\|=\rho(\mathrm{x})^{\mathrm{q}: \mathrm{p}_{\| \mathrm{v}} \|_{M}, \mathrm{v}, \mathrm{w} \varepsilon \mathrm{E} .}
\end{aligned}
$$

LEMMA 3.4. For each $\mathrm{x} \varepsilon \mathrm{G}, \mathrm{v} \varepsilon \mathrm{E}$,

$$
\|\sigma(\mathrm{x}, \mathrm{v})\|=\sup \left\{\rho(\mathrm{y})^{\mathrm{q}: \mathrm{p}}\|\mathrm{w}\|:(\mathrm{y}, \mathrm{w}) \sim(\mathrm{x}, \mathrm{v})\right\}
$$

PROPOSITION 3.5. The bundle $(E, \mathrm{X}, \tau)$ is a Banach bundle (as in section 1 of [6]). 
Let $S(X, E)$ denote the linear space of cross-sections from $X$ into $E$, $\operatorname{CS}(X, E)$ those that are continuous and $\operatorname{CS}_{C}(X, E)$ the continuous cross-sections with compact support. Analogously, recall [2, sec. 4] that there is a continuity structure $\Lambda$ in $\Pi E_{\xi}$ given by $\Lambda=\{\mathrm{Sf}\}$, where

$$
\operatorname{Sf}(\pi(x))=\operatorname{Sf}(x)=\sigma\left(x, \rho(x)^{\left.-1 / q_{f}(x)\right), x \in G,}\right.
$$

for $f$ in $H_{q}(G, L) \cap C(G, E)$, where $H_{q}(G, L)$ is the space of $(L, q)$-homogeneous functions on $G$. (In [2], $\Lambda$ was denoted by $\Lambda_{q^{*}}$ ) As in sections 1 and 5 of [4], we then have the space $C(\Lambda)$ of $\Lambda$-continuous vector fields and the subspace $C_{c}(\Lambda)$ of compactly supported such fields. Note that $S(X, E)=\Pi E_{\xi}$, which in turn is in bijective correspondence with $\mathrm{H}_{\mathrm{q}}(\mathrm{G}, \mathrm{L})$ via the above mapping $\mathrm{f} \rightarrow \mathrm{Sf}$. Also, $C(\Lambda)=\Lambda[2,4.15]$, so that

$$
C(\Lambda)=S\left(H_{q}(G, L) \cap C(G, E)\right)
$$

The continuity structure $\Lambda$ yields a topology (the $\Lambda$-topology) on $E$ making $(E, X, \tau)$ a Banach bundle having the property that the elements of $C(\Lambda)$ are the continuous cross-sections relative to the $\Lambda$-topology [6, Prop. 1.6]. Actually, the space $C(\Lambda)$ is also the space of quotient-continuous cross-sections. [7, sec. 1]

THEOREM 3.6. The quotient and $\Lambda$-topologies are the same.

Before proving this theorem, observe that we then have $\operatorname{CS}(X, E)=C(\Lambda)$ and $\operatorname{CS}_{c}(X, E)=C_{c}(\Lambda)$. Furthermore, if $\mu$ is the measure $d \xi$ on $X$, then $L^{p}(\Lambda, \mu)$ [4, sec. 6] is the same as the space $L^{P}((E, X, \tau), \mu)[6$, sec. 2], which is the ${ }_{L}{ }^{p}$-completion of $\operatorname{CS}_{C}(X, E)$. However, ${ }_{L}^{p}(\Lambda, \mu)$ is isometrically isomorphic to the representation space $F_{U}$ of $U$ and is the representation space $F_{W}$ of $W$. Hence, the representation space of the bundle version of $\mathrm{W}$ will be $\mathrm{L}^{\mathrm{P}}((E, \mathrm{X}, \tau), \mu)$.

PROOF OF 3.6. A basic $\Lambda$-open subset of $E$ is of the form

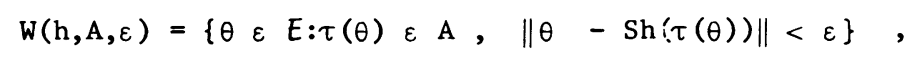

where $h \varepsilon \mathrm{H}_{\mathrm{q}}(\mathrm{G}, \mathrm{L}) \cap \mathrm{C}(\mathrm{G}, \mathrm{E}), \mathrm{A}$ is an open subset of $\mathrm{X}$ and $\varepsilon>0$. Let $\theta=\sigma(x, v)$ be an element of $W(h, A, \varepsilon)$, so that $\tau(\theta)=\pi(x) \varepsilon A$ and 


$$
\begin{aligned}
& \|\theta-\operatorname{Sh}(\tau(\theta))\|=\|\sigma(x, v)-\operatorname{Sh}(x)\| \\
& =\left\|\sigma\left(x, v-\rho(x)^{-1 / q_{h}} h(x)\right)\right\| \\
& =\rho(x)^{q: p_{\| v}}-\rho(x)^{-1 / q_{h}(x) \|_{M}} \\
& <\varepsilon \text {. }
\end{aligned}
$$

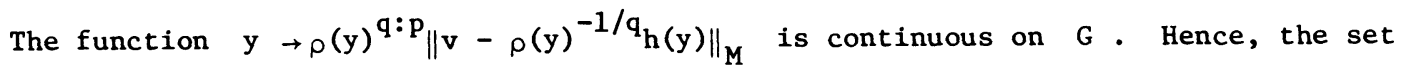
$N$ of $y$ in $G$ where this function is less than $\varepsilon / 2$ is open in $G$. Also, $\mathbf{x} \varepsilon \mathrm{N}$, so that $\mathrm{x} \in \mathrm{N} \cap \mathrm{Zx} \cap \pi^{-1}(\mathrm{~A})$, which is a neighborhood of $\mathrm{x}$ in $\mathrm{G}$. Thus, this set contains an open neighborhood $B$ of $x$. For

$$
a=\max \left\{\rho(y)^{q: p}: y \varepsilon Z x\right\},
$$

define

$$
C=\{w \varepsilon E:\|v-w\|<\varepsilon / 2 a b\} .
$$

Then $C$ is an open subset of $E$ containing $v$. Thus, $\sigma(B \times C)$ is a basic quotient-open neighborhood of $\sigma(x, v)=\theta$ which can be shown to be in $W(h, A, \varepsilon)$. Conversely, let $\sigma(B \times C)$ be a basic quotient-open subset of $E$, for $B$ open in $G$ and $C$ open in $E$. Let $\theta \in \sigma(B \times C)$. Then $\theta=\sigma(x, v)$, for some $\mathbf{x} \varepsilon \mathrm{B}, \mathrm{v} \varepsilon \mathrm{C}$. There exists $\mathbf{r}>0$ such that the ball $\{\mathbf{w} \varepsilon \mathrm{E}:\|\mathbf{w}-\mathbf{v}\|<\mathrm{r} / \mathrm{a}\}$ is contained in C. Choose c sufficiently large so that $c>2 a / m$, where

$$
\mathrm{m}=\min \left\{\rho(y)^{\mathrm{q}: \mathrm{p}}: \mathrm{y} \in \mathrm{Zx}\right\}>0 \text {. }
$$

Since $\left\{h(y): h \in C_{q}(G, L)\right\}$ is dense in $E$ (and hence in $E_{M}$ ) for each $y$ in $G$ $[1,3.8]$ and $h \rightarrow \rho^{-1 / q_{h}}$ is a bijection of $C_{q}(G, L)$, there exists $h$ in $C_{q}(G, L)$ such that

$$
\| v-\rho(x)^{-1 / q_{h}(x) \|_{M}<r / a c}
$$

By the continuity of $h$, there exists an open neighborhood $N$ of $x$ in $G$ such that $\mathrm{N} \subseteq \mathrm{Zx} \cap \mathrm{V}$ and

$$
\| v-\rho(y)^{-1 / q_{h}(y) \|_{M}<r / a c, \quad y \in N}
$$

Let $A=\pi(N)$ and $\varepsilon=r / c$. Then $W(h, A, \varepsilon)$ is a basic $\Lambda$-open neighborhood of $\sigma(x, v)=\theta$ in $E$ which is contained in $\sigma(B \times C)$. Therefore, the two topologies are the same. 


\section{THE KERNEL FIELD BUNDLE}

Our next objective is to construct another Banach bundle for which the kernel fields $\mathrm{K}_{\varphi}, \varphi \in \mathrm{C}_{\mathrm{C}}(\mathrm{G})$, are continuous cross-sections.

Let $\approx$ be the equivalence relation on $\mathrm{G} \times \mathrm{G} \times \operatorname{Hom}(\mathrm{E})$ defined by: $(x, y, T) \approx\left(x^{\prime}, y^{\prime}, T^{\prime}\right)$ if there exist $r, s$ in $H$ such that $x^{\prime}=r x, y^{\prime}=s y$ and $\mathrm{T}^{\prime}=\mathrm{L}(\mathrm{r}) \mathrm{TL}(\mathrm{s})^{-1}$. Let $H$ denote the space of equivalence classes (with quotient topology) and $\alpha: G \times G \times \operatorname{Hom}(E) \rightarrow H$ the canonical projection. Also, let $\beta: H \rightarrow X \times X$ be the (well-defined) projection given by $\beta(\alpha(x, y, T))=(\pi(x), \pi(y))$, $\mathrm{x}, \mathrm{y} \varepsilon \mathrm{G}, \mathrm{T} \in \operatorname{Hom}(\mathrm{E})$. We then have the following composition:

$$
G \times G \times \operatorname{Hom}(E) \stackrel{a}{\rightarrow} \stackrel{\beta}{\rightarrow} \stackrel{x}{\rightarrow} \times X
$$

LEMMA 4.1. The mapping $\alpha$ is continuous and open.

PROOF. The openness of $\alpha$ follows from the fact that the saturation $a^{-1}(a(A \times B \times C))$ of a basic open subset $A \times B \times C$ of $G \times G \times$ Hom $(E)$ is of the f orm

$$
U\left\{r A \times s B \times L(r) C L(s)^{-1}: r, s \varepsilon H\right\}
$$

which is open in $G \times G \times \operatorname{Hom}(E)$.

LEMMA 4.2. The mapping $\beta: H \rightarrow X \times X$ is continuous and open.

PROOF. To see that $\beta$ is open, observe that $\beta(A)=(\pi \times \pi)\left(\alpha^{-1}(A)\right)$ for any subset $A$ of $H$. The lemma then follows from the facts that a is continuous and $\pi \times \pi \quad$ is open.

PROPOSITION 4.3. The space $H$ is Hausdorff.

PROOF. Similar to that of 3.3 .

The next step is to make $(H, X \times X, \beta)$ into a Banach bundle. For $\xi, \eta \varepsilon X$, define $H_{\xi, \eta}=\beta^{-1}(\xi, \eta)$. For fixed $x$ in $\xi$, y in $\eta$, each element of $H_{\xi, \eta}$ is uniquely of the form $a(x, y, T)$, for $T$ in $\operatorname{Hom}(E)$. Define: 


$$
\begin{gathered}
\alpha(x, y, T)+\alpha\left(x, y, T^{\prime}\right)=\alpha\left(x, y, T+T^{\prime}\right), \\
\operatorname{ca}(x, y, T)=\alpha(x, y, c T),
\end{gathered}
$$

and

$$
\|\alpha(x, y, T)\|=(\rho(x) / \rho(y))^{q: p_{\| T}} \|_{M}, T, T^{\prime} \varepsilon \operatorname{Hom}(E), \quad c \varepsilon \mathbb{C},
$$

where the norm is well-defined. Under these operations $H_{\xi, \eta}$ is a Banach space. In fact:

THEOREM 4.4. The space $H_{\xi, \eta}$ is isometrically isomorphic to $\operatorname{Hom}\left(E_{\eta}, E_{\xi}\right)$. PROOF. Fix $a(x, y, T)$ in $H_{\xi, \eta}$ and define $T^{\prime}: E_{\eta} \quad E_{\xi}$ by

$$
\mathrm{T}^{\prime}(\sigma(\mathrm{y}, \mathrm{v}))=\sigma(\mathrm{x}, \mathrm{Tv}), \quad \mathrm{v} \varepsilon \mathrm{E} \text {. }
$$

Then $T^{\prime}$ is well-defined, linear and $\left\|T^{\prime}\right\|=\|a(x, y, T)\|$ as we shall next verify. We have:

$$
\left\|T^{\prime}\right\|=\sup \left\{\left\|T^{\prime}(\alpha(y, v))\right\|: \mathbf{v} \varepsilon E,\|a(y, v)\|=1\right\},
$$

where $\|\sigma(y, v)\|=\rho(y)^{q: p}\|v\|_{M}$. Thus, $\|\sigma(y, v)\|=1$ if and only if $\|v\|_{M}=\rho(y)^{p: q}$. Also,

$$
\begin{aligned}
& \left\|T^{\prime}(\sigma(y, v))\right\|=\|\sigma(x, T v)\| \\
& =\rho(x)^{\mathrm{q}: \mathrm{p}_{\|}}\|\mathrm{T} v\|_{M} \\
& =(\rho(x) / \rho(y))^{q: p_{\| T}} \|(y)^{\left.q: p_{v}\right) \|_{M}} \text {. }
\end{aligned}
$$

Therefore,

$$
\begin{aligned}
& \left\|\mathrm{T}^{\prime}\right\|=\sup \left\{\left\|\mathrm{T}^{\prime}(\sigma(\mathrm{y}, \mathrm{v}))\right\|: \mathrm{v} \varepsilon \mathrm{E},\|\mathrm{v}\|_{\mathrm{M}}=\rho(\mathrm{y})^{\mathrm{p}: \mathrm{q}}\right\} \\
& \left.=\sup \left\{(\rho(x) / \rho(y))^{q: p_{\| T}\left(\rho(y)^{q: P}\right.} \mathrm{p}\right)\left\|_{M}: v \varepsilon E,\right\| v \|_{M}=\rho(y)^{p: q}\right\} \\
& =\sup \left\{(\rho(x) / \rho(y))^{q: p_{\| T}}\|\|_{M}: w \varepsilon E,\|w\|_{M}=1\right\} \\
& =(\rho(\mathbf{x}) / \rho(\mathbf{y}))^{\mathrm{q}: \mathbf{p}}\|\mathbf{T}\|_{\mathbf{M}} \\
& =\|\alpha(x, y, T)\| \text {. }
\end{aligned}
$$

Hence, $T^{\prime} \varepsilon \operatorname{Hom}\left(E_{\eta}, E_{\xi}\right)$ and we have a mapping $a(x, y, T) \rightarrow T^{\prime}$ of $H_{\xi, \eta}$ into $\operatorname{Hom}\left(E_{\eta}, E_{\xi}\right)$, which is clearly a linear isometry. We will be done once we show that this mapping is onto. 
Recall that $E, E_{M}$ and the $E_{\xi}, \xi \varepsilon X$, are all equivalent Banach spaces. Thus, the same is true of $\operatorname{Hom}(E), \operatorname{Hom}\left(E_{M}\right)$ and the $\operatorname{Hom}\left(E_{\eta}, E_{\xi}\right), \xi, \eta \varepsilon X$. In particular, if $T \varepsilon \operatorname{Hom}(E)$, then the corresponding element of $\operatorname{Hom}\left(E_{\eta}, E_{\xi}\right)$ is the operator $T^{\prime}$ which is the image of $a(x, y, T)$ under our identification, $x \varepsilon \xi$, y $\varepsilon \eta$

Consequent1y, modulo the isomorphisms, the field $\left\{\operatorname{Hom}\left(E_{\eta}, E_{\xi}\right): \xi, \eta \varepsilon X\right\}$ of Banach spaces is the same as the field $\left\{H_{\xi, \eta}: \xi, \eta \varepsilon X\right\} \cdot$

REMARK 4.5. Recall that in 3.4 we verified that

$$
\|\sigma(x, v)\|=\sup \left\{\rho(y)^{q: p_{1}}\|w\|(x, v) \sim(y, w)\right\} .
$$

The analogous question here is: does

$$
\|\alpha(x, y, T)\|=\sup \left\{\left(\rho\left(x^{\prime}\right) / \rho\left(y^{\prime}\right)\right)^{q: p_{\|}}\left\|T^{\prime}\right\|: \quad(x, y, T) \approx\left(x^{\prime}, y^{\prime}, T^{\prime}\right)\right\} \quad ?
$$

The answer depends on the question: does

$$
\|\mathrm{T}\|_{\mathrm{M}}=\sup \left\{\left\|\mathrm{M}(\mathrm{r}) \mathrm{TM}(\mathrm{s})^{-1}\right\|: \mathrm{r}, \mathrm{s} \varepsilon \mathrm{H}\right\}, \mathrm{T} \varepsilon \operatorname{Hom}(\mathrm{E}) \quad \text { ? }
$$

We believe the answer to both questions is yes; however, we have been able to only partially verify the latter.

PROPOSITION 4.6. The bundle $(H, X \times X, \beta)$ is a Banach bundle.

As in section 3, we obtain the spaces $S(X \times X, H)$ and $\operatorname{CS}(X \times X, H)$ of crosssections and continuous cross-sections respectively. The space $\operatorname{cs}(x \times x, H)$ is then the space of continuous kernels.

The vector field analogue of this space requires the existence of a continuity structure in $\Pi \operatorname{Hom}\left(E_{\eta}, E_{\xi}\right)$. This was essentially accomplished in section 4 of [2] as follows. Let $H_{L}(G \times G, H o m(E))$ denote the linear space of mappings $B: G \times G \rightarrow \operatorname{Hom}(E)$ satisfying

$$
B(r x, s y)=L(r) B(x, y) L(s)^{-1}, r, s \varepsilon H, x, y \in G \text {. }
$$

For each such $B$, define $R B(\xi, \eta): E_{\eta} \rightarrow E_{\xi}, \xi, \eta \varepsilon x$, by

$$
\mathrm{RB}(\xi, \eta)(\sigma(\mathrm{y}, \mathrm{v}))=\sigma(\mathrm{x}, \mathrm{B}(\mathrm{x}, \mathrm{y}) \mathrm{v}), \mathrm{x} \varepsilon \xi, \mathrm{y} \varepsilon \eta, \mathrm{v} \varepsilon \mathrm{E} \text {. }
$$


Then $\mathrm{RB}(\xi, \eta) \varepsilon \operatorname{Hom}\left(\mathrm{E}_{\eta}, \mathrm{E}_{\xi}\right)$, so that $\mathrm{RB} \varepsilon \Pi \operatorname{Hom}\left(\mathrm{E}_{\eta}, \mathrm{E}_{\xi}\right)$ and we thus have a mapping

$$
R: H_{L}(G \times G, \operatorname{Hom}(E)) \rightarrow \Pi \operatorname{Hom}\left(E_{\eta}, E_{\xi}\right) \text {. }
$$

Note that $R B(\xi, \eta)=B(x, y)^{\prime}$, which is the element corresponding to $a(x, y, B(x, y))$ under the bijection between $H_{\xi, \eta}$ and $\operatorname{Hom}\left(E_{\eta}, E_{\xi}\right)$ (4.4).

LEMMA 4.7. The mapping $R$ is a bijection.

PROOF. The onto property is proved using a (possibly non-measurable) crosssection.

Now consider the linear space

$$
C_{L}(G \times G, \operatorname{Hom}(E))=H_{L}(G \times G, \operatorname{Hom}(E)) \cap C(G \times G, \operatorname{Hom}(E)) .
$$

The set $\Omega=\left\{R B: B \in C_{L}(G \times G, \operatorname{Hom}(E))\right\}$ was observed to be a. precontinuity structure in $\Pi \operatorname{Hom}\left(\mathrm{E}_{\eta}, \mathrm{E}_{\xi}\right)[2,4.32]$. This means that we were unable to verify that $\{R B(\xi, \eta): R B \varepsilon \Omega\}$ is dense in $\operatorname{Hom}\left(E_{\eta}, E_{\xi}\right), \xi, \eta \varepsilon x$. If this is not the case, then the S-topology on $H$ is not the same as the quotient topology - the latter is Hausdorff while the former is not. Moreover, our proof in 3.6 (which we would like to duplicate here) required this density property. This is not a significant problem since we can bypass it by simply restricting our attention to the appropriate portion of $H$. Before doing so, the following result shows to what extent the density property does hold and (more importantly) suggests why it may not hold in general.

THEOREM 4.8. The subspace $\{R B(\xi, \eta): R B \quad \varepsilon \Omega\}$ is strongly dense in $\operatorname{Hom}\left(E_{\eta}, E_{\xi}\right), \xi, \eta \varepsilon X$

PROOF. (After section 3 of [1]). Let $\varphi, \psi$ be elements of $C_{C}(G)$ and $T$ an element of $\operatorname{Hom}(E)$. Define

$$
F(x, y)=\varphi(x) \psi(y) T, \quad x, y \in G .
$$

Then $F: G \times G \rightarrow \operatorname{Hom}(E)$ is continuous and has compact support. For each $x, y$ in $G$, consider the mapping 


$$
(r, s) \rightarrow L(r)^{-1} F(r x, s y) L(s)
$$

of $\mathrm{H} \times \mathrm{H}$ into $\mathrm{Hom}(\mathrm{E})$. Since $\mathrm{L}$ is strongly continuous, so is this mapping. Hence, for $v$ in $E$, the mapping

$$
(r, s) \rightarrow\left[L(r)^{-1} F(r x, s y) L(s)\right](v)
$$

of $\mathrm{H} \times \mathrm{H}$ into $\mathrm{E}$ is continuous and has compact support. Thus, it defines an element $F^{L}(x, y)(v)$ of $E$, where

$$
F^{L}(x, y)(v)=\iint_{H \times H}\left[L(r)^{-1} F(r x, s y) L(s)\right](v) d r d s \text {. }
$$

The mapping $\mathrm{F}^{\mathrm{L}}(\mathrm{x}, \mathrm{y})$ is easily seen to be an element of $\operatorname{Hom}(\mathrm{E})$; in $\mathrm{fact}$, $F^{L} \varepsilon H_{L}(G \times G, \operatorname{Hom}(E))$. Moreover, since $\varphi$ and $\psi$ are uniformly continuous, we actually have that $\mathrm{F}^{\mathrm{L}} \varepsilon \mathrm{C}_{\mathrm{L}}(\mathrm{G} \times \mathrm{G}, \operatorname{Hom}(\mathrm{E}))$. Unfortunately, however, we are able to verify only that $\left\{F^{L}(e, e): F=\varphi \psi T\right\}$ is strongly dense in Hom(E), using the strong continuity of $L$. Hence, $\left\{B(e, e): B \varepsilon C_{L}(G \times G\right.$, Hom $\left.(E))\right\}$ is strongly dense in Hom(E) - By right translating $\varphi$ and $\psi$ by $x$ and $y$ respectively, we see that $\left\{B(x, y): B \varepsilon C_{L}(G \times G, \operatorname{Hom}(E))\right\}$ is strongly dense in $\operatorname{Hom}(E), x, y \varepsilon G$. The theorem then follows from this fact by the definition of $R B$, for $B$ as above. It appears from the previous proof that $\Omega$ may not be a complete continuity structure since $\mathrm{L}$ is not norm-continuous in general. In any event, as we have indicated above, this difficulty is easily circumvented.

LEMMA 4.9. The space $\Omega$ is a continuity structure if and only if $\left\{B(x, y): B \in C_{L}(G \times G\right.$, Hom $\left.(E))\right\}$ is dense in Hom(E) for some (equivalently) all $(x, y) \varepsilon G \times G$.

Let $\operatorname{Hom}_{L}(E)$ denote the norm closure in Hom(E) of the span of the set

$$
\{B(r x, s y): r, s \in H, B \varepsilon \operatorname{Hom}(E)\}
$$

(independent of $x, y)$. Then $G \times G \times \operatorname{Hom}_{L}(E)$ is a saturated subset of $G \times G \times \operatorname{Hom}(E)$, i.e. it is a union of equivalence classes. Let

$$
H_{L}=a\left(G \times G \times \operatorname{Hom}_{L}(E)\right)
$$


and $\beta_{L}=\beta / H_{L}$. Then $H_{L}$ is equipped with the relativized quotient topology of $H$ (or equivalently, the quotient topology of $G \times G \times \operatorname{Hom}_{L}(E)$ ) and $\left(H_{L}, X \times X, \beta_{L}\right)$ is a Banach bundle with

$$
\beta_{L}^{-1}(\xi, \eta) \equiv\left(H_{L}\right)_{\xi, \eta}=H_{L} \cap H_{\xi, \eta}=H_{L} \cap \beta^{-1}(\xi, \eta), \quad \xi, \eta \varepsilon x .
$$

Similarly, we let $\operatorname{Hom}_{L}\left(E_{\eta}, E_{\xi}\right)$ denote the closed subspace of Hom $\left(E_{\eta}, E_{\xi}\right)$ corresponding to $\operatorname{Hom}_{L}(E)$, so that

$$
\operatorname{Hom}_{L}\left(E_{\eta}, E_{\xi}\right) \cong\left(H_{L}\right)_{\xi, \eta}, \xi, \eta \varepsilon x
$$

It is clear that $\Omega$ is a (complete) continuity structure in $\Pi H^{\prime} m_{L}\left(E_{\eta}, E_{\xi}\right) \cong H_{L}$. Consequently, $H_{L}$ is also equipped with the $\Omega$-topology.

THEOREM 4.10. The quotient and $\Omega$-topologies on $H_{L}$ are the same.

PROOF. This is proved in the same way as 3.6 now that $\Omega$ has the density property.

We have thus verified that the continuous cross-sections of the kernel bundle $\left(H_{L}, X \times X, \beta_{L}\right)$ correspond exactly to the $\Omega$-continuous kernel fields in $\Pi \operatorname{Hom}_{L}\left(E_{\eta}, E_{\xi}\right)$. Of course, if $\Omega$ has the desired density property, then the L-subscript may be dropped in 4.10, i.e. $\mathrm{C}(\Omega)=\mathrm{CS}(\mathrm{X} \times \mathrm{X}, \mathrm{H})$ - the ideal result.

\section{THE CONTINUOUS KERNELS}

We are now in a position to complete our project. Recall (section 2) that $I_{\varphi}$ is a continuous mapping of $G \times G$ into $\operatorname{Hom}(E)$. For $\varphi$ in $C_{C}(G)$, define

$$
k_{\varphi}: G \times G \rightarrow G \times G \times \operatorname{Hom}(E)
$$

by

$$
\mathrm{k}_{\varphi}(\mathrm{x}, \mathrm{y})=\left(\mathrm{x}, \mathrm{y}, \rho(\mathrm{x})^{-1 / \mathrm{q}} \rho(\mathrm{y})^{1 / \mathrm{q}_{\mathrm{I}}} \mathrm{I}_{\varphi}(\mathrm{x}, \mathrm{y})\right), \mathrm{x}, \mathrm{y} \in \mathrm{G} .
$$

Then $k_{\varphi}$ is continuous and constant on cosets. Hence, it defines a continuous mapping of $X \times X$ into $G \times G \times H o m(E)$. Furthermore, it follows that the following diagram commutes (modulo isomorphism): 


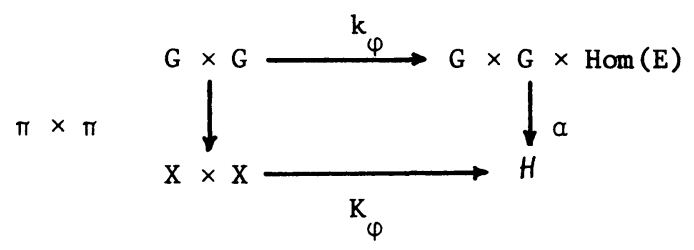

where $\mathrm{K}_{\varphi}(\xi, \eta): \mathrm{E}_{\eta} \rightarrow \mathrm{E}_{\xi}$ is given by $[2, \mathrm{p} .20]$

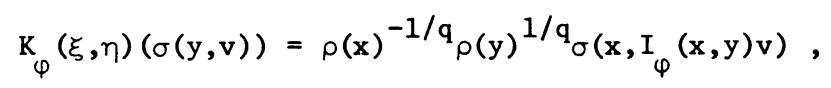

for $\xi, \eta \varepsilon \mathrm{X}, \mathrm{x} \varepsilon \xi, \mathrm{y} \varepsilon \eta, \mathrm{v} \varepsilon \mathrm{E}$. Hence, the kernel $\mathrm{K}_{\varphi}$ for the operator $\mathrm{W}(\varphi)$ is a continuous cross-section. We had already seen [2, 4.33] that it is an $\Omega$-continuous kernel field. Finally, since $\mathrm{K}_{\varphi}=\mathrm{RB}_{\varphi}$, for $\mathrm{B}_{\varphi}=\rho^{-1 / \mathrm{q}} \theta \rho^{1 / \mathrm{q}} \mathrm{I}_{\varphi}$, we see that $\mathrm{K}_{\varphi}: \mathrm{X} \times \mathrm{X} \rightarrow \mathrm{H}_{\mathrm{L}}$, i.e. $\mathrm{K}_{\varphi} \varepsilon \Pi \operatorname{Hom}_{\mathrm{L}}\left(\mathrm{E}_{\eta}, \mathrm{E}_{\xi}\right)$, so that the two notions of continuity for $\mathrm{K}_{\varphi}$ are the same.

In conclusion, let us briefly summarize what we have shown. The induced representation $U$ is isometrically equivalent to a representation $W$ of $G$ on the bundle space $L^{p}((E, X, \tau), \mu)$, where $W(x)$ acts on $\operatorname{CS}_{c}(X, E)$ by right translation by $x, x \in G$. The integrated form of $W$ is given by

$$
\mathrm{W}(\varphi) \mathrm{g}(\xi)=\int_{\mathrm{X}} \mathrm{K}_{\varphi}(\xi, \eta) \mathrm{g}(\eta) \mathrm{d} \eta, \xi \varepsilon \mathrm{X}, \mathrm{g} \varepsilon \mathrm{CS}_{\mathrm{c}}(\mathrm{X}, E),
$$

where $\mathrm{K}_{\varphi}: \mathrm{X} \times \mathrm{X} \rightarrow \mathrm{H}_{\mathrm{L}} \subseteq H$ is a continuous kernel for $\mathrm{W}(\varphi), \varphi \in \mathrm{C}_{\mathrm{c}}(\mathrm{G})$.

of the four ways we have of realizing the integrated form of the induced representation (U,V, together with the vector field and bundle versions of $W$ ), the last one is the most satisfying and usable.

ACKNOWLEDGEMENT. The author was partially supported by an Oakland University Research Fellowship. 


\section{REFERENCES}

1. FONTENOT, R.A. and SCHOCHETMAN, I.E., Induced representations of groups on Banach spaces, Rocky Mtn. J. Math. 7(1977), 53-82.

2. SCHOCHETMAN, I.E., Integral operators in the theory of induced Banach representations, Mem. Amer. Math. Soc., No. 207, 1978.

3. GODEMENT, R. Sur 1a théorie des représentations unitaires, Ann. Math., 53(1951), 68-124.

4. SCHOCHETMAN, I.E., Kernels and integral operators for continuous sums of Banach spaces, Mem. Amer. Math. Soc., No. 202, 1978.

5. FELL, J.M.G., Induced representations and Banach*-algebraic bundles, Lect. Notes Math., No. 582, 1977.

6. FELL, J.M.G., An extension of Mackey's method to Banach*-algebraic bundles, Mem. Amer. Math. Soc., No. 90, 1969.

7. SCHOCHETMAN, I.E., Generalized group algebras and their bundles, submitted. 